\title{
Modeling the Effect of Chemotaxis on Glioblastoma Tumor Progression
}

\author{
Francisco G. Vital-Lopez and Antonios Armaou \\ Dept. of Chemical Engineering, The Pennsylvania State University, University Park, PA 16802 \\ Michelle Hutnik \\ Medical Science Dept., The Angiogenesis Foundation, Cambridge, MA 02238 \\ Costas D. Maranas \\ Dept. of Chemical Engineering, The Pennsylvania State University, University Park, PA 16802
}

DOI 10.1002/aic.12296

Published online June 22, 2010 in Wiley Online Library (wileyonlinelibrary.com).

Tumor progression depends on the intricate interplay between biological processes that span the molecular and macroscopic scales. A mathematical agent-based model is presented to describe the 3-D (three-dimensional) progression of a brain tumor type (i.e., glioblastoma multiforme) as the collective behavior of individual tumor cells whose fate is determined by intracellular signaling pathways (i.e., MAPK pathway) that are governed by the temporal-spatial distribution of key biochemical cues (i.e., growth factors, nutrients). The model is used to investigate how tumor growth and invasiveness depend on the response of migrating tumor cells to chemoattractants. Simulation results suggest that individual cell sensitivity to chemical gradients is necessary to generate in silico tumors with the irregular shape and diffusive tumor-stroma interface characteristic of glioblastomas. In addition, vascular network damage influences tumor growth and invasiveness. The results quantitatively recapitulate the central role that nutrient availability and signaling proteins have on tumor invasive properties. (ㅇ 2010 American Institute of Chemical Engineers AIChE J, 57: 778-792, 2011

Keywords: glioblastoma, modeling of tumor dynamics, signaling in tumor growth, 3-D tumor morphology

\section{Introduction}

The American Cancer Society estimated that during 2008, 21,810 adults would be diagnosed with malignant brain or spinal cord tumors in the US, with 13,070 patients dying due to their disease (cancer.org, accessed January 18, 2009). The most common adult malignant brain tumor is glioblastoma multiforme (grade IV in the World Health Organization classification). Glioblastomas are intracranial neoplasms characterized by uncontrolled proliferation, and generally exhibit a necrotic core, marked angiogenesis, asymmetrical infiltrating invasiveness and are highly refractory to radio/chemother-

\footnotetext{
Correspondence concerning this article should be addressed to C. D. Maranas at costas@psu.edu.

(C) 2010 American Institute of Chemical Engineers
}

apy. Current glioblastoma treatments include supportive care to alleviate symptoms of the disease (e.g., cerebral edema, seizures, cognitive dysfunctions, etc.), and local and/or systemic therapies to ablate the tumor. Antitumor therapies traditionally involve surgical resection followed by radiotherapy and chemotherapy. Recent clinical trials have demonstrated that advances in imaging, surgical and radiotherapy techniques, coupled with sequential or concurrent combinations of chemotherapies and/or targeted therapies have resulted in improvements in response rate and progression free survival. ${ }^{1-5}$ However, almost all glioblastoma patients relapse after initial therapy and the median overall survival is about 15 months, only modestly improving over the last 25 years. ${ }^{6}$ A major factor in treatment failure is the diffuse infiltration of highly invasive tumor cells into the surrounding tissue from the early stages of tumor development, generally 
resulting in recurrence just a few months after surgery. ${ }^{6,7}$ Considerable efforts have been directed to elucidate the underlying mechanisms of the perivascular migration of cancer cells at the cellular ${ }^{8-10}$ and tumor ${ }^{11}$ levels using in vitro experiments. Observations of different cell lines of glioblastoma suggest that the directionality of their migrating paths could be a determinant factor in the invasiveness of the tumors. ${ }^{12}$

A quantitative understanding of invasiveness requires that tumor cell migration be investigated in concert with cellular proliferation, necrosis, apoptosis, host vessel co-option and angiogenesis. These processes span multiple time and spatial scales. At the cellular level, tumor cell phenotype and migratory behavior is determined by its local environment. Tumor cells require a minimum level of nutrients to thrive, whereas the transduction of signaling cues regulates their phenotype (i.e., migratory or proliferative). It has been observed that the growth factor-induced phosphorylation of a downstream component of the MAPK signaling pathway (i.e., ERK) correlates with the migratory and proliferative behavior of tumor cells. ${ }^{13}$ The MAPK signaling pathway can be triggered by several different growth factors, including $\mathrm{TGF} \alpha$, that bind to the epidermal growth factor receptor (EGFR), ${ }^{14}$ which is amplified (in $40 \%$ of cases) or over expressed (in 50\% of cases) in gliomas. ${ }^{15,16}$ At the tumor level, the progression of the tumor is determined by the spatiotemporal distribution of nutrients and signaling cues. Nutrients and bloodborn growth factors (i.e., TGF $\alpha$ ) that trigger the activation of the MAPK signaling pathway are supplied by the vasculature. In addition, TGF $\alpha$-stimulated tumor cells produce TGF $\alpha$, closing an autocrine circuit. Moreover, the migrating direction of tumor cells is determined by the distribution of chemoattractants (e.g., nutrients). The tumor dynamically alters the distribution of nutrients and signaling cues due to an increase in the metabolic demand and the remodeling of the vasculature (i.e., vessel occlusion). During the initial stage of tumor progression, tumor growth is considered "avascular" and does not require the formation of new blood vessels. Very aggressive glioblastomas have been found to depend on host vessel co-option for tumor growth and can be angiogenesis independent. ${ }^{17}$ In this work, angiogenesis is not considered.

In this article, we introduce a mathematical model to investigate how the response of tumor cells to biochemical gradients affects the paths of migrating cells, and, hence, the invasiveness and morphology of glioblastomas. We model tumor progression as the outcome of the evolution in space and time of a collection of tumor cells that dynamically interact with their environment. The model integrates the dynamics of key biological processes occurring at the cellular and tumor levels. The cellular level component for each individual tumor cell is determined by a set of rules that govern the phenotype and migration of tumor cells. These rules assume that the tumor cell phenotype depends on the concentration of nutrients and the activation of the MAPK signaling pathway. The tumor level component of the model determines the spatiotemporal distribution of key biochemical cues such as oxygen (as a representative nutrient) and $\mathrm{TGF} \alpha$. A relatively high-resolution determination of these distributions is important for a better assessment of their effect on the tumor progression. For this purpose, we construct a complex vascular network that resembles the geometry and functionality of the vasculature of the white matter of the brain. The proposed model produces 3-D tumors with a relatively large number of cells (in the order of
$10^{6}$ ) nourishing from a realistic vasculature as compared with other current agent-based models that produced tumors with cell populations in the order of $10^{4}$ and simplified nutrient sources (e.g., point sources). Simulation results demonstrate that different tumor cell responses to chemical gradients result in markedly different tumor morphologies and invasion rates. The next subsection provides an overview of previous results on mathematical modeling in cancer research and particularly of brain tumors. Subsequently, we present the details of the mathematical model and its implementation accompanied by the simulation results. Finally, we discuss the implications of the obtained results and highlight future research directions.

\section{Mathematical modeling of tumor progression}

Mathematical modeling of tumor progression has received considerable attention over the last years. Here we only provide a brief recount of the major modeling frameworks used as well as representative results; this is by no means a comprehensive list. The reader may refer to reviews focused on modeling of tumor growth, ${ }^{18,19}$ tumor-induced angiogene$\operatorname{sis}^{20,21}$ and glioblastomas ${ }^{22-24}$ for more information. The simplest models of tumor development and related bioprocesses assume that the system is homogeneous. This allows encoding the dynamics of key system components (e.g., cell populations and proteins levels) into a set of ordinary differential equations (ODEs). This framework has been extensively used for describing intracellular processes (e.g., signaling pathways and regulatory networks). ${ }^{25-28}$ It has also been used to simulate the temporal evolution of tumor cell populations and the effect of therapeutic agents. ${ }^{27,29-35}$ However, solid tumors develop in a highly heterogeneous environment, and a more realistic description of their progression requires a spatially distributed representation leading to partial differential equations (PDEs). Not surprisingly, the new spatial dimension of tumor development captured by the PDEs comes at the expense of higher computational requirements and the need of efficient solution algorithms. There are a number of proposed modeling frameworks relying on PDE descriptions. In some efforts, populations of cells are described as continuous fields and are generally deterministic. ${ }^{1,36-50}$ Although this abstraction enables us to capture the effects of spatial variability of select factors on tumor progression, the incorporation of intracellular mechanisms that determine cellular behavior remains problematic.

Multiscale cellular automaton or agent-based models explicitly couple intracellular mechanisms with description of tumor level processes. Generally in these models, the concentration of nutrients and growth factors are treated as continuous fields whereas cells are considered as discrete entities governed by a set of rules representing their intracellular processes and intercellular interactions. Such rules commonly take the form of algebraic expressions, logical expressions and/or random processes that depend on the variables that define the state of the extracellular environment. ${ }^{51-55}$ In more elaborate frameworks, the rules themselves are models of the intracellular pathways that control cell fate, in the form of ODEs. ${ }^{56,57}$ Similarly, hybrid continuous-discrete models have been used to couple angiogenesis with tumor growth. ${ }^{58-60}$ Other models that consider cells as discrete entities are based on biomechanical principles. Examples of 
these models include Potts models, in which cellular growth, deformation and movement are described based on systemenergy reduction; ${ }^{61-63}$ macroscopic models of solid tumors that consider cell adhesion; ${ }^{64}$ immersed boundary methods with distributed sources use to describe growth and division of single cells. ${ }^{65}$ The two main computational difficulties associated with the solution of agent-based models arise from the large number of tumor cells present within the simulation domain and the solution of PDEs in 3-D domains. Methods to alleviate the computational burden related with the number of cells have been proposed. In a multiresolution approach, tumor cells are classified into spatial clusters reducing the number of rule evaluations. However, its application to 3-D simulations has not been published. An additional complication (associated with the large number of cells) results when the model accounts for mechanical factors influencing tumor development.

In the context of brain tumors, the growth and invasion of gliomas, both in vitro and in vivo experimental settings, have been studied using distributed continuous models $^{11,36,47,66}$ and the cellular automata framework. ${ }^{55,56}$ The latter framework has also been applied to analyze the effect of a dynamic vasculature on tumor growth. ${ }^{54,60,67}$ Distributed continuous models have also been used to determine effective regimes of therapeutic treatments such as radiotherapy and chemotherapy to inhibit tumor growth. ${ }^{42,43}$ The dynamics of tumor evolution have also been integrated with flow dynamics of interstitial fluid to investigate the effect of pressure gradients on the transport of a chemotherapeutic agent released from an implanted polymer after surgery. ${ }^{68}$

\section{Proposed model}

In this article, we develop a multiscale agent-based model to describe tumor growth and invasion resulting from the proliferation and migration of individual tumor cells under biologically relevant conditions. The model consists of two interdependent component describing processes at the cellular and tumor levels. At the cellular level, the state of individual tumor cells is governed by a set of rules depending on their local environment (i.e., concentrations of nutrients and $\mathrm{TGF} \alpha$ ) and intracellular signaling pathways (i.e., MAPK signaling pathway). The tumor level component determines the spatiotemporal distribution of the key biochemical cues. The two components are connected through the interchange of information required to solve the whole model. Specifically, the local concentration of biochemical cues for every tumor cell is obtained from the solution of the tumor level component whereas the production and consumption terms for the tumor level component are determined by cellular level component. The details of the tumor and cellular level components of the model are presented in the following subsections.

Tumor Level. The tumor level model captures the spatiotemporal distribution of oxygen, TGF $\alpha$ and tumor cells within the simulation domain. The profiles of the chemical species are described by a set of PDEs. Tumor cells are treated as agents dwelling in a regular square grid and can migrate or proliferate only into empty lattice sites. The details of the simulation domain and the PDEs are given in the next subsections, followed by the explanation of the rules that govern the tumor cell agents.

Simulation Domain. Glioblastomas may arise in any part of the central nervous system and are frequently found in the white matter of the brain. ${ }^{69}$ The simulation domain $(\Omega)$ is a cubic region of the white matter of dimension $12 \times 12 \times 12$ $\mathrm{mm}^{3}$, and it consists of two subdomains. The tumor progression subdomain, defined as a cube of dimension $4 \times 4 \times 4$ $\mathrm{mm}^{3}$, is at the center of $\Omega$ and it is surrounded by a buffer region (the rest of the domain). The buffer region is included to minimize the effect of the boundary conditions on chemical species concentrations. During the simulation, we record the spatiotemporal distribution of oxygen and TGF $\alpha$ as well as the state of every tumor cell. The state of tumor cells is defined by their phenotype, location, cellular mass and the activation level of their MAPK pathway (i.e., phosphorylation level of ERK $\left(\right.$ ERK $\left._{\mathrm{act}}\right)$ ).

Vascular Network. We constructed a vascular network with a structure and functionality similar to that of the white matter. Nonaka et al. $^{70}$ using soft X-ray and diaphanized specimens of normal adult brains found that large arteries run straight through the white matter toward the lateral ventricle. The arteries have lateral branches with tree-like structures that connect to venules through capillary vessels. We constructed the vascular network in an iterative process as shown in Figure 1. Briefly, seeds for arteries and veins were randomly placed on the upper face of the simulation domain and were grown adding a vessel segment along the gradient of oxygen concentration until the vessels reached the bottom face. Subsequently, elements of three vessel segments in the form of "Y" were added along the gradient of the oxygen concentration at the tips of the current network. Capillary vessels were added to connect arteries with veins when the distance between nodes was less than $200 \mu \mathrm{m}$, each node has less than three vessels, and the radius of those vessels was less than $20 \mu \mathrm{m}$. The radius of the vessels was calculated using Murray's rule $r_{p}^{a}=r_{s 1}^{a}+r_{s 2}^{a}, \alpha=2.7,{ }^{71}$ where $p$, $s 1$ and $s 2$ indicate the parent and the children vessels, respectively. The oxygen concentration was computed by solving a PDE similar to the one described in the next subsection. Initially, it was assumed that all current vessel segments supply oxygen. Once arteries and veins have connected, it was assumed that only vessel segments that belong to a path connecting an artery with a vein can supply oxygen. The network was grown until the average concentration reached the average oxygen concentration in brain tissue $\left(0.022 \mathrm{mM}^{72}\right)$. The resulting vascular network has approximately 310,000 vessel segments. Figure 1 shows the vascular network in the tumor progression domain.

Although we recognize that brain tumors alter blood vessel morphology, ${ }^{73}$ in vivo models have shown that aggressive glioblastomas can co-opt the host vasculature and grow without signs of angiogenesis. ${ }^{17}$ In this article, we assume that the tumor remodels the vasculature by occluding the vessels as it grows. In the absence of a detailed mechanical model, we assume that vessels are occluded when tumor cells occupied a fraction (ranging from $v o_{\text {min }}=0.1$ for the smallest vessels to $v o_{\max }=0.5$ for the largest vessels) of their original vessel volume. Furthermore, we assume that only proliferative cells can overtake a lattice site occupied by an active vessel. In the absence of a detailed mechanistic description, 

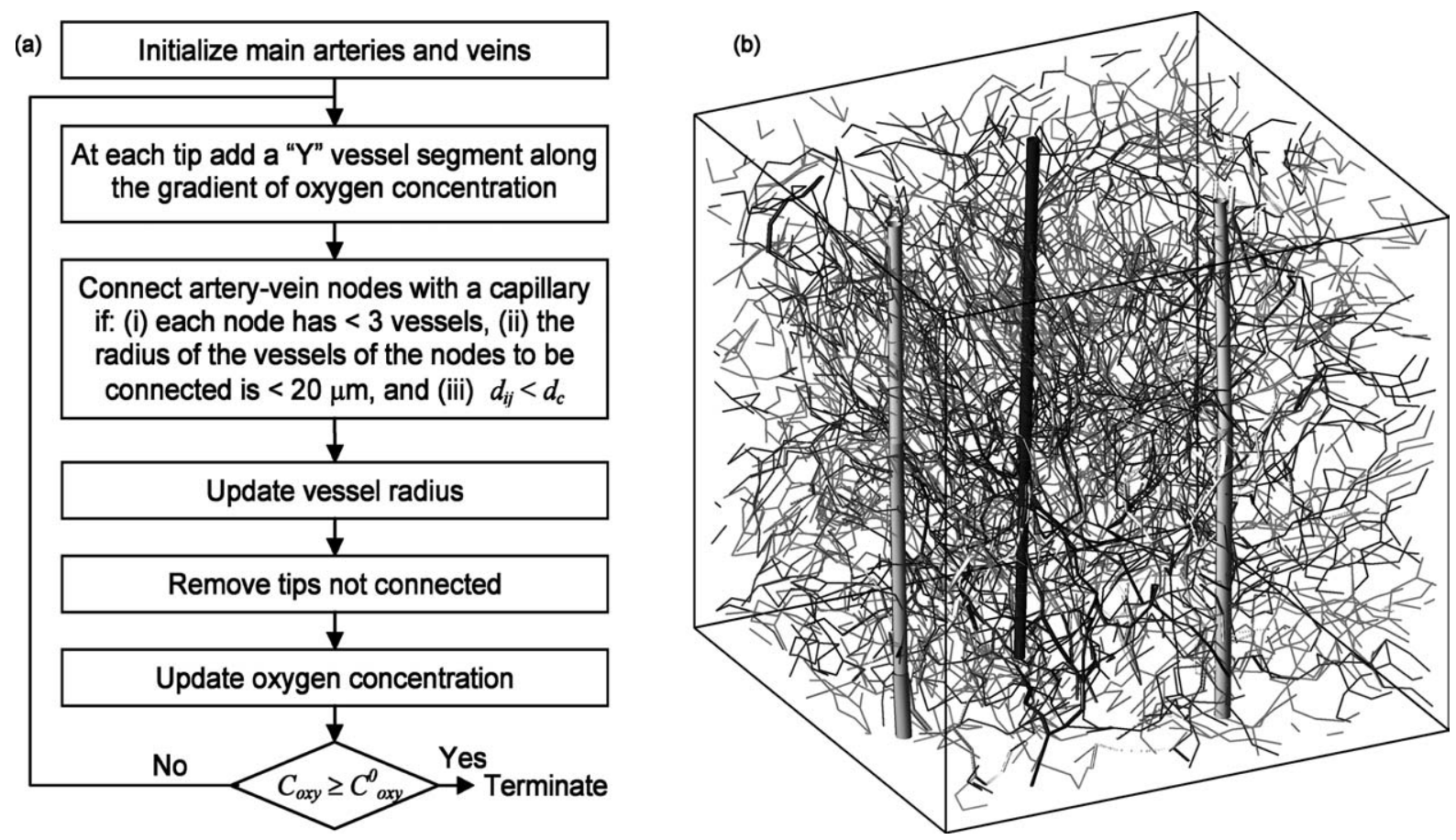

Figure 1. Vascular network used in the simulations.

(a) Algorithm to construct the vascular network. $d_{c}$ is the maximum distance between tips to add a capillary and $d_{i j}$ is the distance between tips $i$ and $j$. (b) vascular network within the tumor progression subdomain. The black and white largest vessels are arteries and veins, respectively. Vessels of small radius are not shown.

we assume that the probability of a tumor cell to take a lattice site of a vessel is one tenth of the probability of proliferating into a free-lattice site. When a vessel is occluded, it is removed from the network together with all the vessels that are no longer part of a path between an artery and a vein due to the disruption.

Spatiotemporal Distribution of Oxygen and TGF $\boldsymbol{\alpha}$. Oxygen and TGF $\alpha$ concentrations are considered to be continuous fields described by a set of PDEs

$$
\begin{aligned}
\partial_{t} O & =\nabla \cdot\left(D_{O}(t, z) \nabla O\right)+K_{T}^{O}(t, z)\left(O_{v}-O\right) \\
& -k_{O}(t, z) O, \quad O \in \Omega \\
\partial_{t} T & =\nabla \cdot\left(D_{T}(t, z) \nabla T\right)+K_{T}^{T}(t, z)\left(T_{v}-T\right) \\
& -k_{T}(t, z) T+S\left(t, z, C_{\text {intra }}\right), \quad T \in \Omega,
\end{aligned}
$$

with boundary and initial conditions

$$
\begin{aligned}
& \mathbf{n} \cdot\left(D_{O}(t, z) \nabla O=0, \quad O \in \Gamma, \quad O(t=0)=O_{S S},\right. \\
& \mathbf{n} \cdot\left(D_{T}(t, z) \nabla T=0, \quad T \in \Gamma, \quad T(t=0)=T_{S S},\right.
\end{aligned}
$$

where $O$ is the extracellular oxygen concentration, $\partial_{t}$ denotes the partial derivative with respect to time, $D_{o}$ is the oxygen diffusion coefficient, $K_{T}^{O}$ is the supply rate of oxygen from the blood vessels, and $k_{o}$ is the oxygen consumption rate constant. $T$ represents TGF $\alpha$ extracellular concentration, and the parameters in the respective conservation equation are analogous to the ones discussed for oxygen. $\Omega$ is defined as the computational domain of the PDE and $\Gamma$ is the boundary of $\Omega$, whereas $\mathbf{n}$ is the normal vector to $\Gamma$ and $O_{S S}, T_{S S}$ are the steady-state concentrations of oxygen and TGF $\alpha$, respectively.
$S(\cdot)$ refers to production term of $\mathrm{TGF} \alpha$ by tumor cells. Boundary conditions determining the concentration or the fluxes of the extracellular species are required for properly solving the PDEs. However, such boundary conditions are not available. To circumvent this limitation, we extended the simulation domain to include tissue far away from the tumor, thus, creating a buffer region, and then assumed no-flux boundary condition.

The parameters of the tumor level model were collected from the open literature when available or estimated to approximate reported levels of the chemical species considered in the brain (Table 1). Typical values for oxygen and TGF $\alpha$ concentrations are $0.022 \mathrm{mM}^{72}$ and $2.7 \times 10^{-2} \mathrm{nM}^{56}$ respectively. The oxygen consumption rate and the $\mathrm{TGF} \alpha$ degradation rate were set to give a diffusion length of 150 $\mu \mathrm{m}$ and $400 \mu \mathrm{m},{ }^{74}$ respectively. The supply rate of oxygen and TGF $\alpha$ for the vessels is estimated such that the average oxygen and $\mathrm{TGF} \alpha$ concentrations match typical values on the brain. Similarly, considering the hypercellularity glioblastomas $^{75}$ and that migrating and proliferating tumor cells consume about 2-5 times more resources than quiescent cells, ${ }^{62,76}$ we assume that the oxygen consumption rates for tumor cells are higher than the surrounding tissue by a factor of two for quiescent cells, and by a factor of four for migrating and proliferating cells. Finally, we postulate that blood flow is blocked when tumor cells compress the blood vessels, thus, ceasing to be a source of nutrients as will be described in the subsection entitled Migrating direction.

Cellular Level. The cellular level model consists of a set of rules governing the behavior of tumor cells. In brief, tumor cell phenotype depends on the activation level of the MAPK pathway and the availability of nutrients, whereas 
Table 1. Parameters for the Tumor Level Model

\begin{tabular}{|c|c|c|c|c|}
\hline Parameter No. & Parameter & Value (Units) & Description & Reference \\
\hline 1 & $D_{O}$ & $8 \times 10^{-5}\left(\mathrm{~cm}^{2} \mathrm{~s}^{-1}\right)$ & Diffusion coefficient of oxygen & 1 \\
\hline 2 & $K_{T}^{O}$ & $1.25 \times 10^{-1}\left(\mathrm{~cm} \mathrm{~s}^{-1}\right)$ & Supply rate for oxygen & \\
\hline 3 & $k_{O}$ & $1.75 \times 10^{-4}\left(\mathrm{mM} \mathrm{s}^{-1}\right)$ & Consumption rate of oxygen & \\
\hline 4 & $O_{v}$ & $0.07(\mathrm{mM})$ & Oxygen concentration in the blood & 2 \\
\hline 5 & $D_{T}$ & $5.5 \times 10^{-11}\left(\mathrm{~cm}^{2} \mathrm{~s}^{-1}\right)$ & Diffusion coefficient of TGF $\alpha$ & 1 \\
\hline 6 & $k_{T}$ & $6 \times 10^{-5}\left(\mathrm{~s}^{-1}\right)$ & Degradation rate of $\mathrm{TGF} \alpha$ & \\
\hline 7 & $K_{T}^{T}$ & $6.5 \times 10^{-5}\left(\mathrm{~cm} \mathrm{~s}^{-1}\right)$ & Supply rate for $\mathrm{TGF} \alpha$ & \\
\hline 8 & $T_{v}$ & $2.7 \times 10^{-1}(\mathrm{nM})$ & TGF $\alpha$ concentration in the blood & \\
\hline 9 & $g_{1}$ & $1.57 \times 10^{-3}\left(\mathrm{nM} \mathrm{s}^{-1}\right)$ & Production rate of $\mathrm{TGF} \alpha$ & 3 \\
\hline 10 & $K_{M}^{O}$ & $0.37(\mathrm{mM})$ & Kinetic parameter for $\mathrm{TGF} \alpha$ production & \\
\hline $11 ; 12 ; 13$ & $a_{O, Q: M ; P}$ & $2(4,4)$ & Factor of oxygen consumption rate by quiescent/migrating/proliferating cells & \\
\hline 14 & 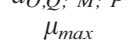 & $2.2 \times 10^{-5}\left(\mathrm{~s}^{-1}\right)$ & Maximum growth rate of tumor cell & \\
\hline 15 & $C_{\text {mass }}^{\text {ave }}$ & 2 (arbitrary units) & Average cellular mass & \\
\hline 16 & $C_{m a x s}^{\operatorname{mass}}$ & 8 (arbitrary units) & Maximum cellular mass & \\
\hline 17 & $h_{n}$ & $0.0033(\mathrm{nM})$ & Threshold for a cell to become necrotic & 4 \\
\hline 18 & $s_{n}$ & 200 (dimensionless) & Steepness of necrotic probability curve & \\
\hline 19 & $P_{Q M}$ & 0.2 & Maximum probability of a quiescent cell to become migrating & \\
\hline 20 & $h_{Q M}$ & $90(\mathrm{nM})$ & Threshold for a quiescent cell to become migrating & \\
\hline 21 & $s_{Q M}$ & $0.14\left(\mathrm{nM}^{-1}\right)$ & Steepness of quiescent to migrating probability curve & \\
\hline 22 & $P_{Q P}$ & 0.1 & Maximum probability of a quiescent cell to become proliferating & \\
\hline 23 & $h_{O P}$ & $100(\mathrm{nM})$ & Threshold for a quiescent cell to become proliferating & \\
\hline 24 & $s_{Q P}$ & $0.14\left(\mathrm{nM}^{-1}\right)$ & Steepness of quiescent to proliferating probability curve & \\
\hline 25 & $P_{M M}$ & 0.9 & Maximum probability of a migrating cell to remain migrating & \\
\hline 26 & $h_{M M}$ & $90(\mathrm{nM})$ & Threshold for a migrating cell to remain migrating & \\
\hline 27 & $s_{M M}$ & $0.14\left(\mathrm{nM}^{-1}\right)$ & Steepness of migrating to migrating probability curve & \\
\hline 28 & $P_{M P}$ & 0.05 & Maximum probability of a migrating cell to become proliferating & \\
\hline 29 & $h_{M P}$ & $100(\mathrm{nM})$ & Threshold for a migrating cell to become proliferating & \\
\hline 30 & $s_{M P}$ & $0.14\left(\mathrm{nM}^{-1}\right)$ & Steepness of migrating to proliferating probability curve & \\
\hline 31 & $v o_{\min }$ & 0.1 & Minimum fraction of vessel volume for occlusion to occur & \\
\hline 32 & $v o_{\max }$ & 0.5 & Maximum fraction of vessel volume for occlusion to occur & \\
\hline 33 & $E R K_{\text {tot }}$ & $3 \times 10^{2}(\mathrm{nM})$ & Total concentration of ERK & 3 \\
\hline 34 & $r_{c}$ & $1 \times 10^{-3} \mathrm{~cm}$ & Nominal tumor cell radius & \\
\hline 35 & $r_{v}$ & $5 \times 10^{-3} \mathrm{~cm}$ & Nominal blood vessel radius & \\
\hline
\end{tabular}

the migration direction depends on the response of migrating cells to nutrient gradients. These rules are described in the following subsections.

MAPK Signaling Pathway. The model of the MAPK signaling pathway determines the TGF $\alpha$-induced activation level of ERK and the amount of autocrine TGF $\alpha$ produced. Figure 2 illustrates the signaling cascade considered in the model. The MAPK signaling pathway model includes 17 species participating in 22 transformations described by either mass-action kinetics or Michaelis-Menten kinetics. The MAPK signaling pathway model is represented by a set of ordinary differential equations

$$
\begin{aligned}
& d_{t} C_{\text {intra }, i}=f_{i}\left(C_{\text {extra }}, C_{\text {intra }}\right), \quad i=1, \ldots, N, \\
& C_{\text {intra }, i}(t=0)=C_{\text {intra }, i}^{0} \quad i=1, \ldots, N,
\end{aligned}
$$

where $d_{t}$ is the derivative with respect to time, $C_{\text {intra }}$ denotes the concentration of the intracellular species, and $f_{i}$ is the righthand side function of the $i^{\text {th }}$ ODE of the system that describes the intracellular dynamics of the MAPK pathway. $C_{\text {intra }}^{0}$ represents the initial concentration of the intracellular species of a given cell. $C_{\text {intra }}^{0}$ is determined by the concentrations for the same cell at the end of the previous iteration. The detailed mathematical expressions and the kinetic parameters can be found in Maly et al. ${ }^{77}$ and are not presented here for brevity reasons. We assume that the production rate of TGF $\alpha$ depends on the metabolic state (i.e., oxygen level) of the tumor cells. Accordingly, we modified the source term for the production of growth factors from the model of Maly et al. ${ }^{77}$ as follows

$$
s=\left(g_{1} \frac{E R K_{a c t}}{E R K_{\text {tot }}+E R K_{a c t}} \frac{O / O_{\max }}{K_{M}^{O}+O / O_{\max }}\right)
$$

where $E R K_{\text {act }}$ is the intracellular concentration of activated ERK. The parameters of this expression are given in Table 1.

\section{Cell phenotype}

Depending on the local nutrient concentrations, the availability of space and the activation level of their MAPK pathway, tumor cells can be necrotic or express the quiescent, migrating or proliferating phenotypes. In our model, this is determined by a stochastic decision process (Figure 3 ). We first check if the tumor cells are viable given their local nutrients concentrations. If this is not the case, the tumor cells become necrotic. This step models tumor cell death by necrosis due to the lack of oxygen. We assume that the probability of tumor cell death is a function of the oxygen concentration (Figure 4a). The probability of tumor cell death is set at 0.5 if the oxygen concentration is reduced at $15 \%$ of its normal level. ${ }^{78}$ If the tumor cells become necrotic then they neither consume nutrients nor produce TGF $\alpha$; even though the production of growth inhibitors by hypoxic cells and waste by viable cells could be important, it currently is not accounted for. 


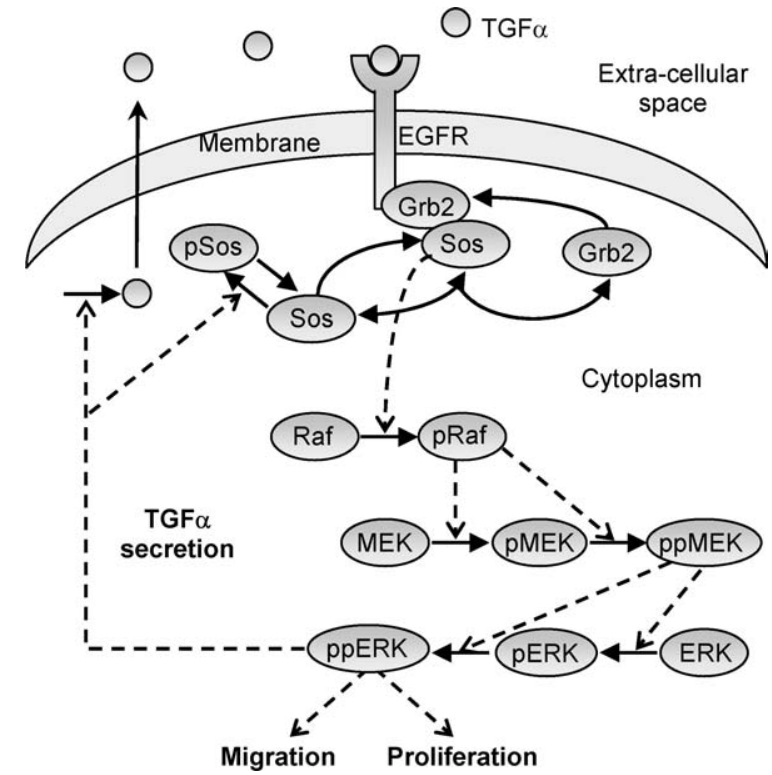

Figure 2. MAPK signaling pathway.

TGF $\alpha$-induced ERK activation determines tumor cells phenotype (i.e., quiescent, migrating or proliferating) and TGF $\alpha$ autrocrine circuit.

Subsequently, we check if viable tumor cells are inhibited due to contact with other tumor cells. ${ }^{78}$ This is based on the assumption that tumor cells proliferate until a maximum cellular density is reached. Subsequently, proliferation occurs only to compensate for cell death and/or cell migration. This assumption has been frequently used in grid constrained agent based models. ${ }^{52,78,79}$ Specifically, tumor cells can proliferate or migrate only if there is a free-lattice site in their neighborhood (i.e., not occupied by another cancer cell), otherwise they become quiescent. If a free-lattice site is available, then we determine the phenotype of the tumor cells. The adoption of different phenotypes by tumor cells is governed by a set of rules that are not fully elucidated. Consequently, modelers have so far relied on empirical rules. Here, we used experimental observations that correlate the level of growth factors (and, therefore, of the activation of the MAPK pathway) with the migration and proliferation rates of astrocytomas cell lines in vitro. ${ }^{80}$ Based on these observations, we model the decision mechanism as a random process depending on the strength of the ERK activation. Briefly, Giese et al. ${ }^{80}$ observed that as astrocytoma cells were stimulated with increasing concentrations of grow factors, migration increases faster than proliferation, but at high concentrations of grow factors proliferation had a considerable increment, whereas migration decreased. Assuming that ERK activation correlates with the concentration of growth factors, our decision process assigns a high probability for tumor cells to become (or remain) quiescent under low levels of $E R K_{\text {act }}$, whereas it favors migration and proliferation for medium and high levels of $E R K_{\text {act }}$, respectively. Furthermore, the probability of quiescent cells becoming proliferating or migrating is also affected by level of nutrients. We assume that tumor cells that acquire the migrating phenotype will preferentially retain this phenotype since active migration suppresses cell proliferation, ${ }^{80}$ but they can nevertheless assume other phenotypes. Proliferating cells, however, retain this phenotype unless they become necrotic or quiescent (when undergoing mitosis or by contact inhibition). Figure $4 \mathrm{~b}$ provides the quantitative rules adopted for these transitions. Finally, we check if proliferating cells undergo mitosis. We assume that mitosis occurs with a probability that depends on the cellular mass (Figure 4c). The probability of mitosis is set at 0.5 when the cellular mass is twice the nominal cellular mass. The cellular mass is calculated assuming cellular growth described by a logistic equation with a growth rate depending on the oxygen concentration as follows

$$
\begin{aligned}
& C_{\text {mass }}=\frac{C_{\text {mass }}^{0} e^{\mu t}}{\left(C_{\text {mass }}^{0} / C_{\text {mass }}^{\max }\right)\left(e^{\mu t}-1\right)+1}, \\
& \mu=\mu_{\max } \frac{O / O_{\max }}{K_{M}^{O}+O / O_{\max }},
\end{aligned}
$$

where $C_{\text {mass }}^{0}$ and $C_{\text {mass }}^{\max }$ are the initial and maximum cellular mass, $\mu$ is the growth rate, and $t$ is the time. $\mu_{\max }$, and $O_{\max }$ are the maximum values of growth rate and oxygen $(O)$ concentration, respectively. The parameters were estimated such that the cellular mass increases from 1 to 2 (arbitrary units) in $24 \mathrm{~h}$ for $O=0.022 \mathrm{nM}$, in agreement with experimental observations for glioblastomas. ${ }^{81}$

Migrating Direction. In vivo, the migration direction of tumor cells is determined by multiple interdependent processes, including, but not limited to chemotaxis, haptotaxis and mechanical forces. Chemotaxis is the directed cell motility along gradients of chemical attractants (e.g., nutrients), or repellents (e.g., metabolic waste). Haptotaxis is the directed cell motility along a positive gradient of adhesion molecules in the extracellular matrix. As an example, in vivo glioma tumors preferentially migrate along white matter tracts and blood vessels. ${ }^{82}$ The quantitative contribution of various processes to the migration mechanism is unknown. For simplicity, our model considers chemotaxis as the exclusive mechanism governing tumor cells migration direction. Since

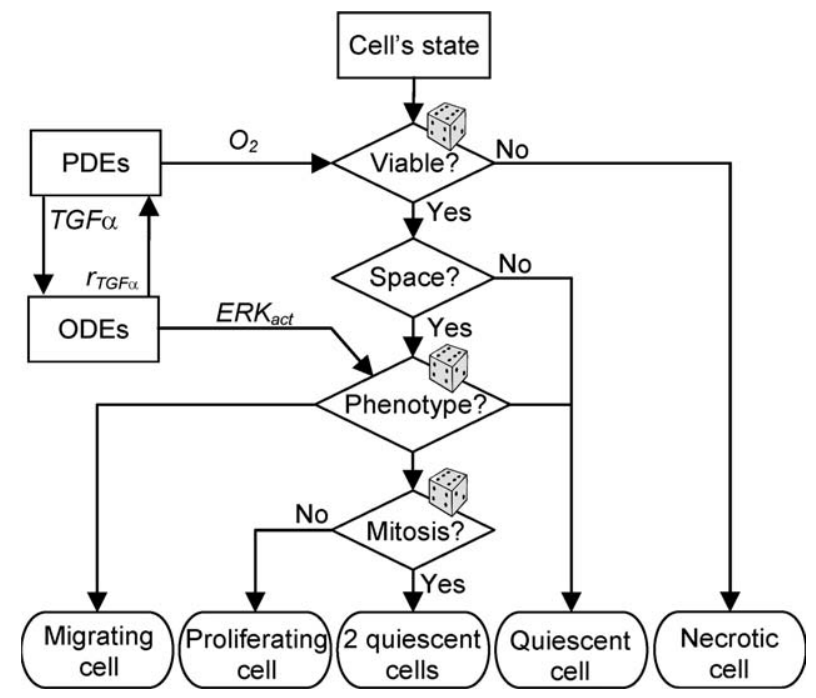

Figure 3. Phenotype transitions tumor cells.

Dice indicate stochastic processes. 

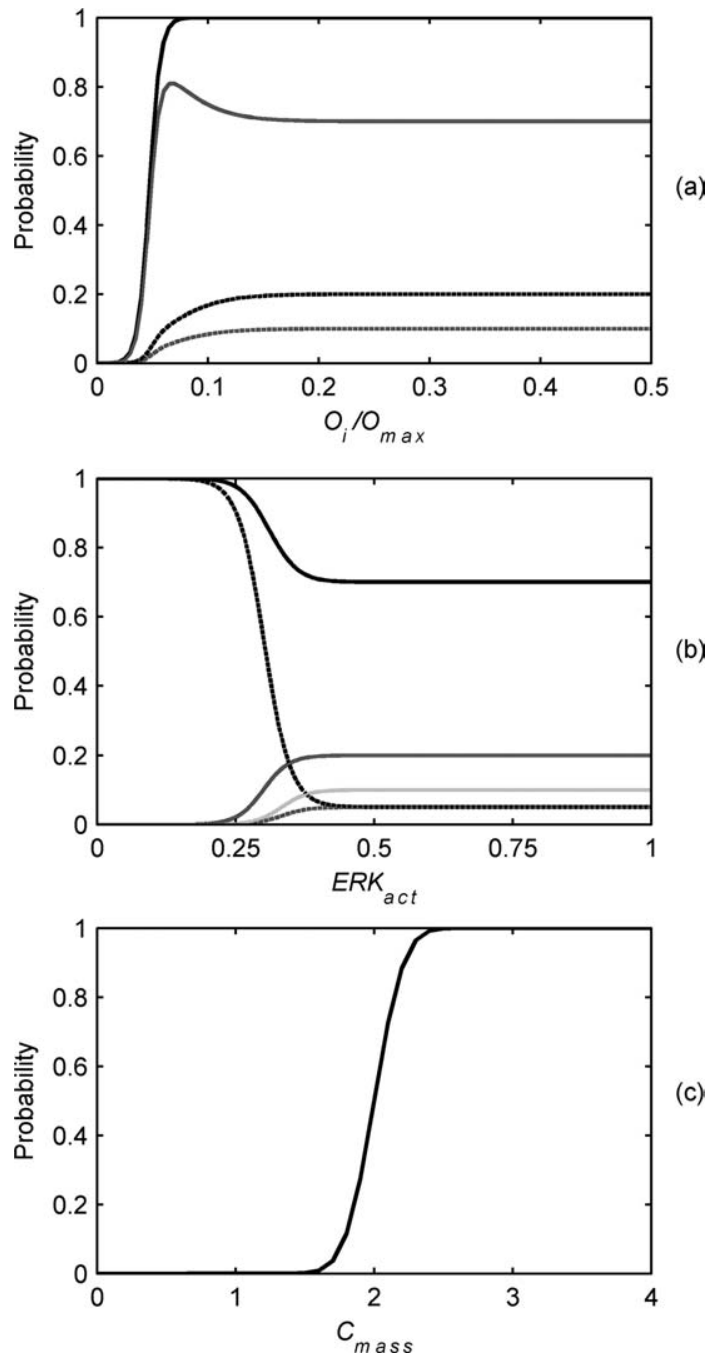

Figure 4. Probabilities of tumor cells for phenotype transitions.

(a) Probability of a tumor cell to remain alive (solid black), become quiescent (solid gray), migrating (dash black) or proliferating (dash gray) as a function of the local oxygen concentration for $E R K_{\mathrm{act}}=1$, (b) probability of a quiescent cell becoming migrating (solid gray) or proliferating (solid light gray) and of a migrating cell becoming quiescent (dashed black) or proliferating (dashed gray) as a function of the activation level of ERK for $O=O \max$, and (c) Probability of a proliferating cell to undergo mitosis as a function of its cellular mass.

the list of chemoattractants (or repellents) affecting chemotaxis for glioblastoma cells is extensive (i.e., growth factors, nutrients, waste products, etc.), further simplifications are necessary. Our model includes only oxygen gradients as the primary factors influencing chemotaxis. This assumption can be considered reasonable as is largely accepted ${ }^{83}$ that nutrient gradients are key chemoattractants for glioblastoma cells.

In the absence of a mechanistic description of chemoattraction, we model the selection of the migrating direction by tumor cells as a stochastic process dependent on local gradients. We assume that tumor cells can move only to a free neighboring lattice site with a probability parameterized by the oxygen level

$$
P_{i}=\frac{h_{i} v_{i}\left(O_{0}+w_{i}\left(O_{i}-O_{0}\right)\right)}{\sum_{j} v_{j}\left(O_{0}+w_{j}\left(O_{j}-O_{0}\right)\right)},
$$

where $P_{i}$ is the probability of a tumor cells to move to a neighboring lattice site $i . O_{0}$ is defined as $O / O_{\max }$ at the current lattice site and it is similarly for $O_{i}$ and $O_{j}$ at sites $i$ and $j$. The index $j$ in the summation represents only the free-lattice sites. $h_{i}$ are weighting factors that take into account the length of the displacement (see Figure 5a). The weights $v_{i}$ characterize the sensitivity of the migrating tumor cells to the nutrient gradients. We considered three different sets of $v_{i}$ to investigate the effect of this mechanism on the tumor morphology. These sets of $v_{i}$ approximate the complete range of sensitivity in accordance with the observed migratory behavior of human glioma cells. ${ }^{12}$ The migratory response corresponding to each set of $v_{i}$ is designated as low, medium and high chemotaxis (see Figure 5b). In the low chemotaxis case (i.e., all $v_{i}=1$ ), migrating tumor cells have low sensitivity to chemoattractant gradients. These cells can move in directions of decreasing nutrient level, resulting in migratory behavior similar to the biased random walk. ${ }^{58}$ In contrast, migrating tumor cells distinguish between negative and positive nutrient gradients in the medium chemotaxis case (i.e., $v_{i}=1$ for free-lattice sites with higher nutrient concentrations than the current position, and $v_{i}=0$ otherwise). Finally, the high chemotaxis case models extreme sensitivity to chemoattractant gradients, resulting in certain migration toward the direction of the highest nutrient concentration increase (i.e., only the direction of highest nutrient concentration has nonzero weight $v=1$ ).

Simulation Algorithm. The simulation is initiated at steady state with the tissue consisting of only normal cells. At $t=0$, a small core of cancer cells (of 5 cells of radius) is introduced at the center of the simulation domain and an iterative integration scheme as shown in Figure 6 is initiated. At every time step $\Delta t$, the tumor level (PDE) model is solved (assuming pseudo steady state) to determine the oxygen and TGF $\alpha$ concentration profiles. These concentrations then become inputs to the cellular level model of every tumor cell. The MAPK signaling pathway model is then integrated for every tumor cell to determine the ERK activation and the TGF $\alpha$; production rate, which in turn become inputs to the PDEs in the next iteration. The phenotype of every cell is then determined depending on the level of oxygen and ERK activation and the position of cells and spatial dependent parameters are updated. The integration proceeds in time until a tumor cell enters the buffer region of the simulation domain or a prespecified time limit is reached.

The implementation of the simulation algorithm poses two challenges that tax memory usage and computational time tractability limits. The first challenge is the solution of the PDEs. A popular method to solve PDEs is the multigrid (MG) method. ${ }^{84}$ In brief, in the MG method the PDE is discretized with different mesh sizes to optimize the convergence rate of relaxation techniques. The foundation of the method is that a considerable fraction of the low-frequency components of a fine mesh are mapped into high frequency nodes on a coarser mesh. We solved the PDEs using a Vcycle MG with 4 levels. The finest level had mesh size of $20 \mu \mathrm{m}$ (the size of a tumor cells) resulting in linear system with $8 \times 10^{6}$ unknowns for the tumor progression domain. 
a

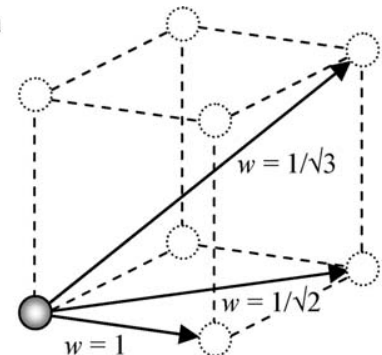

b

Chemotaxis level
Low
$v_{i}=1 \quad \forall i$
Medium $\quad$\begin{tabular}{lllll|}
1 & if $O_{i} \geq O_{0}$ \\
0 & Otherwise &
\end{tabular}
High
$v_{i}=\left\{\begin{array}{llllll}1 & \text { if } O_{i}=\max _{j}\left(O_{j}\right) \\
0 & \text { Otherwise }\end{array}\right.$

Figure 5. Migrating direction parameters.

(a) Weights $w$ account for the distance to neighboring lattice sites, and (b) sets of $v_{i}$ and possible movements of a migrating cell for different levels of chemotaxis.

The coarsest level had a mesh size of $160 \mu \mathrm{m}$ and was solved using a conjugate gradient method. The second challenge is the integration of the MAPK signaling pathway model for a large number of cells (in the order of $10^{6}$ ). We applied the in situ adaptive tabulation (ISAT) method ${ }^{85,86}$ to remedy the difficulties associated with the large number of ODEs evaluations. The basic idea of this method is to approximate the integration of the MAPK signaling pathway model for a given initial condition using previously stored evaluations of a relatively close initial condition. The simulation algorithm was implemented using MATLAB (The MathWorks, Inc., Natick, MA). CPU times for the 3-D simulations were in the order of $10-15 \mathrm{~h}$ in a Pentium D CPU $3.00 \mathrm{GHz}$ with $2 \mathrm{~GB}$ of RAM computer.

\section{Simulation results}

We performed 3-D simulations to investigate the effect of different response scenarios of migrating cells to chemoattractant gradients on tumor morphology and progression. Subsequently, we carried out simulations to assess the combined effect of the response level to chemotaxis and vascular network degeneration on tumor growth dynamics. We also simulated tumors with mixed populations to assess their interactions. Finally, we performed a sensitivity analysis. Three simulations were performed for each study.

\section{Effect of chemotaxis response on tumor progression}

We first compare tumors constructed by tumor cells guided by different levels of chemotaxis under vascular network degeneration conditions. The effect of the chemotaxis level on tumor morphology can be observed in Figure 7. The snapshots of the tumors show the compact core and the invasive edge of the tumor at several times. The compact core (invasive edge) is defined as the tumor regions where more than $95 \%(<95 \%)$ of the lattice sites are occupied by tumor cells. Tumor cells driven by a low level of chemotaxis (LC cells) lead to a regular, compact, spherical shaped tumor core surrounded by a relatively thin invasive edge, which is only slightly affected by the distribution of the biochemical cues (Figure 7a). In the case of tumor cells driven by medium level of chemotaxis (MC cells), the compact core of the tumor assumes an irregular shape resulting from the development of separated regions of high-tumor cell density. High-cellular density spots arose mainly nearby larger vessels as the compact core growing along the large artery at the center of the domain in Figure $7 \mathrm{~b}$, day 30. This dependency on the location of the blood vessels is even more marked for the tumor formed by tumor cells driven by high chemotaxis (HC cells) as can be seen in Figure 7c. In this case, offshoots of the invading edge grow along blood vessels until these are occluded (Figure 8). Subsequently, the tumor offshoots advance toward nearby active vessels. The diffuse nature and irregularity of the tumor surface increases with the level of chemotaxis. The tumor of LC cells has a well-defined boundary. On the other hand, the tumor of MC cells has a diffuse interface although it is relatively uniform

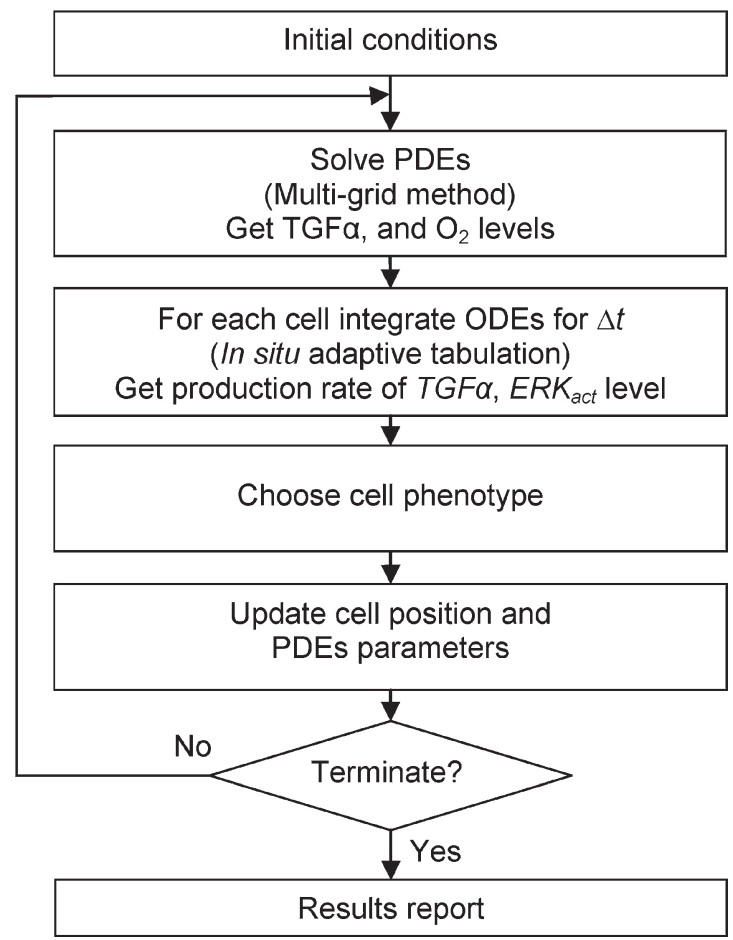

Figure 6. Algorithm to simulate tumor progression. 


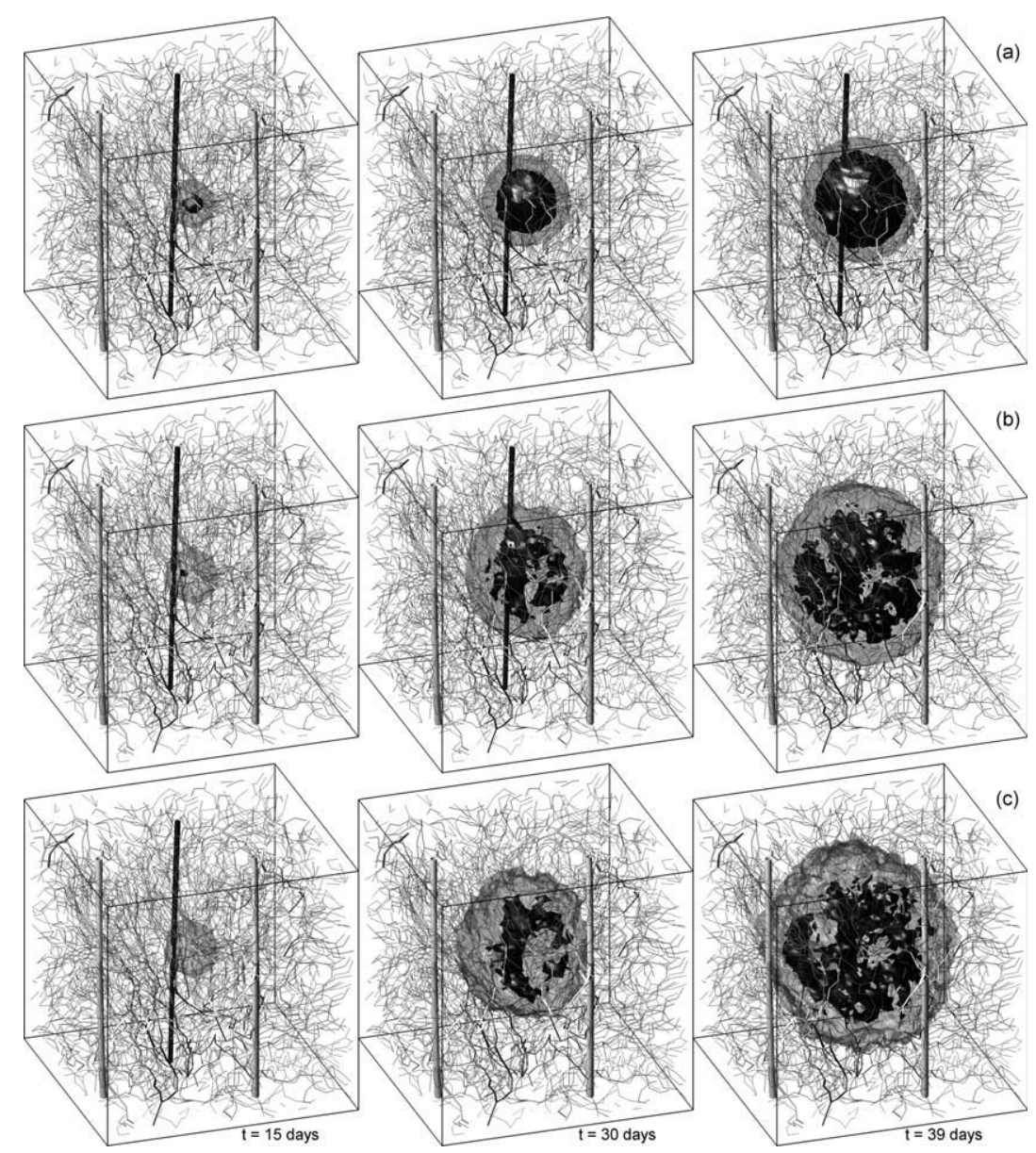

Figure 7. Simulation of tumor progression in the 3-D simulation domain.

Snapshots of the tumors at different times for (a) low, (b) medium, and (c) high chemotaxis. The gray isosurfaces represent the invasive edge of the tumor. The black isosurfaces enclose the compact core of the tumor.

along the compact core. In the high-chemotaxis case, the tumor exhibits a more diffuse and irregular interface.

The level of chemotaxis also affects the tumor invasion rate. At early stages, the growth dynamics of the three tumors is similar. However, the tumor of $\mathrm{MC}$ cells is slightly more proliferative, whereas the tumor of HC cells expands faster than the other tumors. The difference on the invasion rate is more evident at later times as can be seen in Figure 9a. Clearly, HC cells leads to a higher invaded volume compared to that of tumors of LC and MC cells. High chemotaxis also results in tumors with lower average tumor cell density across the compact core of the tumor (Figure 9b). However, the local tumor cell density in the tumors of $\mathrm{MC}$ and $\mathrm{HC}$ cells can be as high as for the tumors of LC cells. The lower average cellular density in these cases is due in part to the presence of noninvaded regions (mostly necrotic) enclosed by the tumor. The faster invasive rim of these tumors destroys the vessels in its path, provoking a drop in the nutrients levels and giving no time for the inner tumor cells to fill up all the space before they become necrotic. Note that lower cellular density entails a lower local metabolic burden which is reflected on a thicker rim of viable cells surrounding the necrotic core (Figure 9b).

\section{The effect of vasculature remodeling on tumor progression depends on the chemotaxis level}

Simulation results in the previous subsection indicate that the effect of chemotaxis on the morphology and growth dynamics of the tumor is strongly connected with the way tumors perturb their environment. We compare tumors simulated using the same conditions as in the previous subsection except that the tumors do not occlude the vascular network. Figure 10 shows the invaded volume of the simulated tumors at day 39. The tumor of LC cells that does not occlude vessels has a larger volume than its counterpart, whereas the effect is reversed for the tumor of $\mathrm{HC}$ cells. The reason for these opposite results is related to the distribution of the growth factor and the different response of the tumor cells to the chemoattractants. Tumors that destroy the vasculature develop large necrotic cores whereas most of the tumor cells remain alive in their counterparts. Since only alive tumor cells produce $\mathrm{TGF} \alpha$, the tumors that do not occlude vessels have higher levels of ERK activation and, consequently, larger fraction of migrating and proliferating cells. This condition combined with the insignificant response to chemoattractant gradients results a higher invasion rate of tumors of LC cells in the case of no vascular network degeneration. The advantage of higher ERK activation is overcome by 


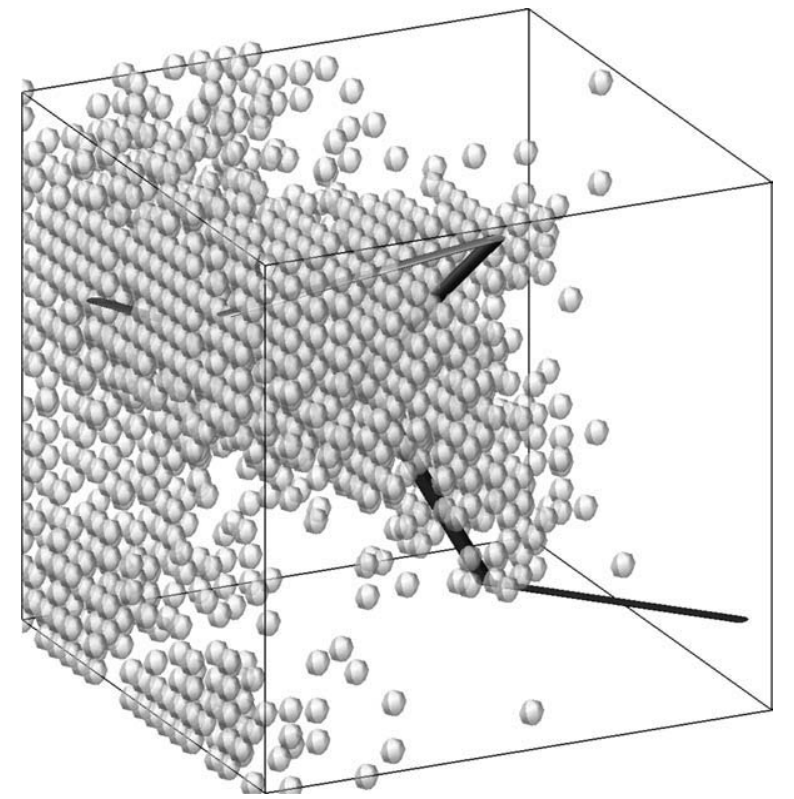

Figure 8. Vessel co-option in simulation of a tumor of HC cells.

effect of the changes on oxygen distribution for the tumors of $\mathrm{HC}$ cells. Most of the $\mathrm{HC}$ cells cluster tightly along the nonoccluded vessels with fewer tumor cells on regions away from the vessels. Oxygen gradients toward the standing vessels also increase the local cellular density, and, consequently, diminishing the spaces for proliferative cells. In the case of tumors of MC cells, these factors cancel out each other resulting in similar invaded volume, although the morphology of the tumors differs. Tumors that occlude vessels have a necrotic core and a more diffuse and uniform invasive edge, whereas their counterparts have offshoots along the vessels. This invasion pattern resembles vascular cooption, a mechanism important in brain tumor growth where tumor cells can infiltrate the vascular bed, leading to a lower density of blood vessels compared to normal tissue. ${ }^{17,87}$

\section{HC cells dominate in tumors of mixed populations by inducing higher rate of death for the other cells}

A common feature of tumors is the heterogeneous genotype and phenotype of their populations. We carried out simulations starting with a tumor seed composed of equal populations of LC, MC and HC cells to study the growth dynamics of the tumor. The time evolution of the number of cells of each type for tumors composed of single and mixed populations is presented in Figure 11a. The populations of MC and $\mathrm{HC}$ cells have reached a larger size in the mixed population tumors than in the single-population tumors at the end of the simulations. This result is a consequence of the lower rate of cell death in the mixed-population tumors (only up to day 26 for MC cells) than in the single population tumors (Figure 11c), whereas the proliferation rate is very similar in both cases (Figure 11b). On the contrary, at early stages the population of LC cells had significant higher proliferation rate in the mixed population tumor than in the single-popula- tion case (Figure 11b). However, the LC cells also had a considerable higher rate of death in the mixed-population tumor that leads to the death of most of these cells at latter stages (Figure 11c). The early increased proliferation of LC cells in the mixed-population tumors is consequence of their higher level of ERK activation (because they are located on the compact core where the TGF $\alpha$ concentration is higher), and the increased availability of space to proliferate created by the dispersion of MC and $\mathrm{HC}$ cells. However, the LC cells cannot move fast enough to escape from the necrotic region front induced by the occlusion of vessels by the MC and $\mathrm{HC}$ cells. In fact, the rate of death of MC cells also became higher for the mixed than for the single-population tumor after day 26 (Figure 11c). Clearly, HC cells dominated the mixed-population tumor at the end of the simulation. However, the main causes of this outcome are the occlusion of the vessels and the difference in the invasion rates rather than the competition of the different type of cells for space.

\section{Sensitivity analysis}

We performed local sensitivity analysis to assess the effect of small changes of the parameter values on the results presented in the previous subsections. The parameters 1 to 32 listed in Table 1 were changed (one at the time), by either increasing or decreasing each by $10 \%$ of its nominal value
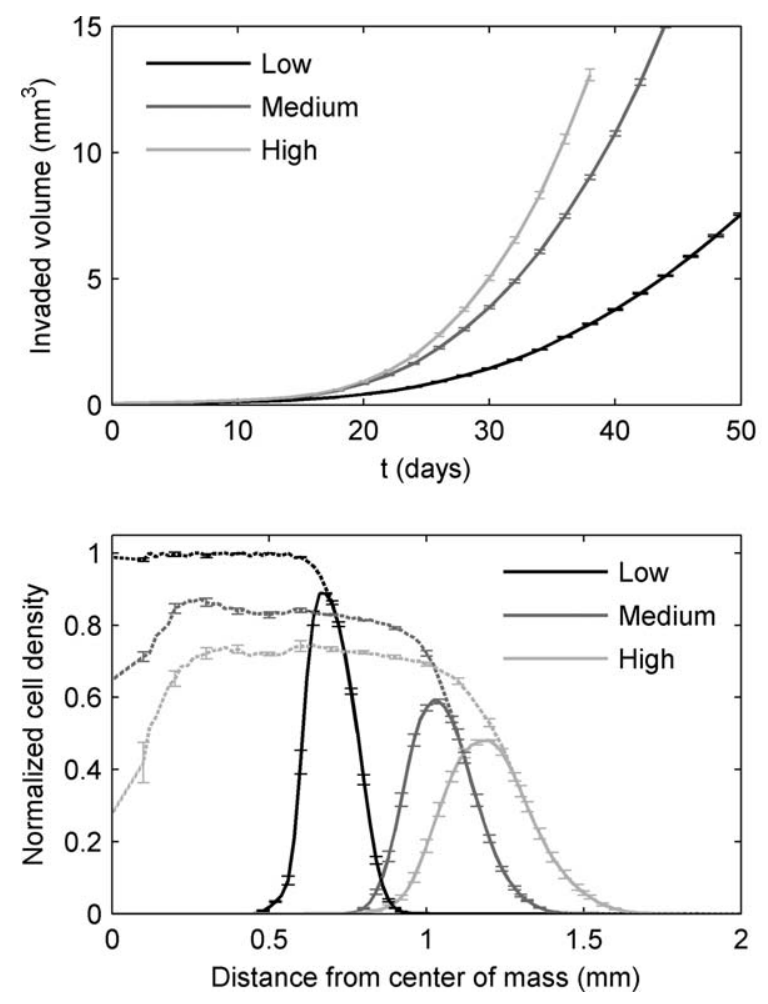

(b)

Figure 9. Temporal profiles of tumor progression when tumors occlude vessels.

(a) Volume invaded by the tumors, and (b) Average tumor cell density of the tumors as a function of the distance from the center of mass of the tumor. Solid lines indicate the density of live tumor cells. Dashed lines indicate the total tumor cell density including necrotic cells. 


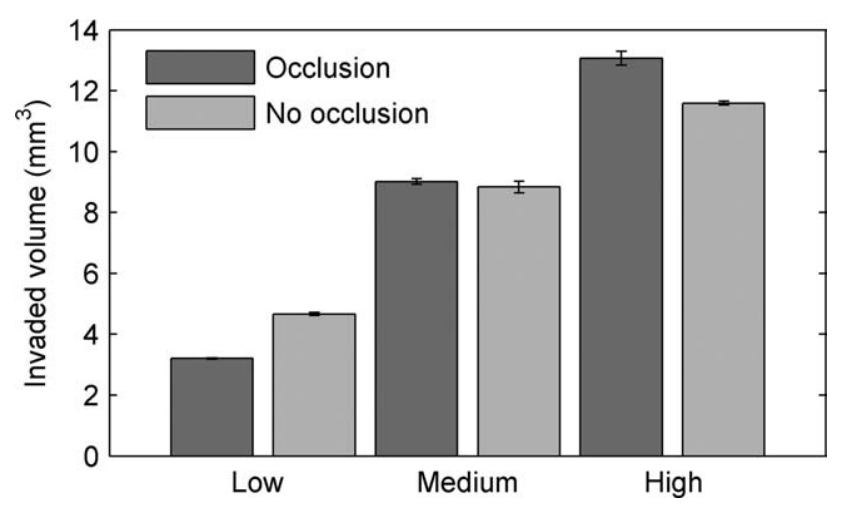

Figure 10. Invaded volume by the tumor with and without vessel occlusion at day 39.

and simulated the model to determine the invaded volume (the parameters of the signaling pathway and the vasculature were not considered). The deviation of the invaded volume from the nominal case gives a measure of the effect of the change in a parameter. Figure 12a shows the deviation of the invaded volume for the MC tumors at day 33 (the final simulation time of the shortest simulation). The largest deviation was caused by changes in the threshold of $E R K_{\text {act }}$ $\left(h_{Q P}, 23\right)$, required for a quiescent cell to acquire the proliferating phenotype. Increasing this parameter by $10 \%$ resulted in an invaded volume of only $4 \%$ of the nominal case due to a low activation of the TGF $\alpha$ autocrine circuit. For the same reason, the second most influential parameter is the production rate of TGF $\alpha$ by tumor cells $\left(g_{1}, 9\right)$. Changes in the consumption rate of oxygen by normal cells $\left(k_{o}, 3\right)$ caused the third largest deviation on the invaded volume. Note that the consumption rates for tumor cells were considered to be factors $\left(a_{O, Q}, a_{O, M}, a_{O, P}, 11,12,13\right)$ of the normal oxygen consumption rate $\left(k_{o}, 3\right)$. When these factors were changed individually, the invaded volume changed up to a less extent. Interestingly, both decreasing and increasing the threshold of $E R K_{\text {act }}$ for quiescent cells to become migrating $\left(h_{Q M}, 20\right)$ resulted in lower invaded volume. For lower $h_{Q M}$, the tumor has a larger fraction of dispersed migrating cells at the expense of the proliferative population at early stages, slowing down the activation of the TGF $\alpha$ autocrine circuit. On the other hand, higher $h_{Q M}$ resulted in smaller tumor due to higher contact inhibition. It should be emphasized that these results correspond to a single-time point, whereas the dependence of the invaded volume in the parameters is dynamic. The effect of changes on the five most dominant parameters on the relative invaded volume for the different levels of chemotaxis is shown in Figure 12b. Even though the changes in these parameters caused large deviations on the invaded volume, the ratio of the invaded volume of the $\mathrm{MC}$ and $\mathrm{HC}$ cases to the $\mathrm{LC}$ case remains very similar to the nominal case with the exceptions of the decrement on $h_{Q P}$, and the increment of $h_{Q M}$. This indicates a complex relation (at least for the choice of parameter values) between the phenotype decision process of quiescent cells and their sensitivity to chemoattractants to determine tumor invasion rate and suggest further studies for better understanding this process.

\section{Summary and Discussion}

We analyzed the effect of the response of migrating tumor cells to chemoattractant gradients on the morphology, growth dynamics and invasive rate of brain tumors. For this purpose, we developed a hybrid multiscale model to simulate tumor progression in a complex vascular network with structure and functionality mimicking that observed in the white matter that enables the determination of more realistic concentration profiles using experimentally derived parameter values. We found that either medium- or high-level chemotaxis is needed to simulate tumors with irregularly shaped necrotic core surrounded by a rim of viable tumor cells and diffuse infiltrations characteristic of glioblastoma macroscopic morphology. ${ }^{7,15}$ This suggests that glioblastoma tumors possess a robust mechanism for preferential growth toward sources of nutrients.

Directional migration (i.e., medium and high chemotaxis) increases tumor growth and invasion rates. Clearly, the faster invasive edge is a direct consequence of migrating tumor cells moving more consistently toward fresh sources of nutrients than in the low-chemotaxis case. Higher response to chemoattractants also contributes to the faster growth of
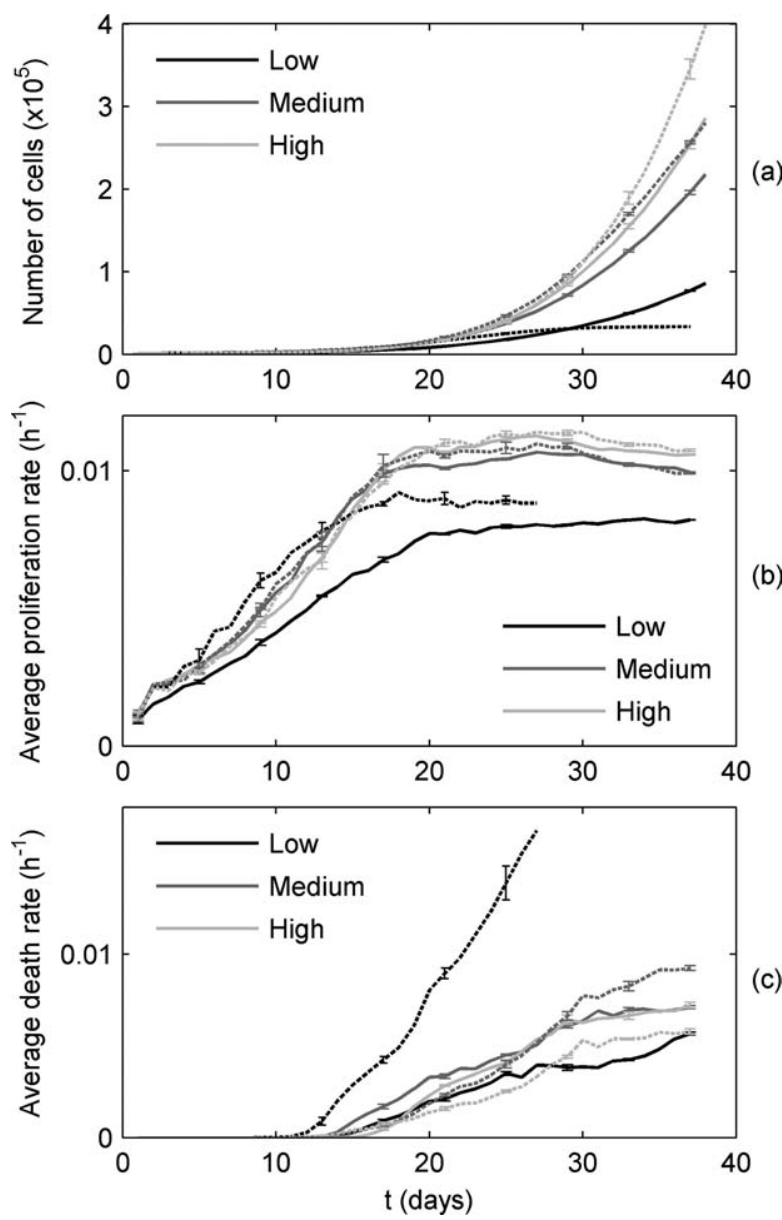

Figure 11. Growth dynamics of tumors of mixed populations.

Time evolution of (a) population size, (b) proliferation rate, and (c) rate of death for each cell type in tumors of mixed and single populations. 

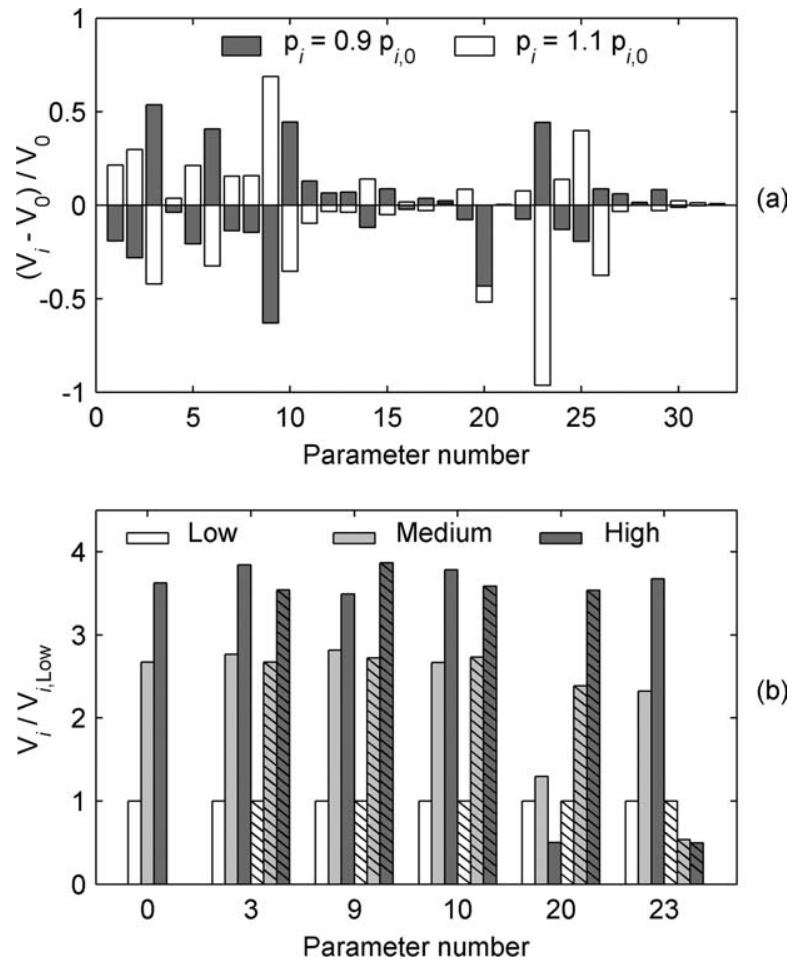

Figure 12. Results of the sensitivity analysis.

(a) Deviations of invaded volume for changes in the individual parameters for the $\mathrm{MC}$ tumors at day $33 . \mathrm{V}_{0}$ and $\mathrm{V}_{i}$ are the invaded volume for the nominal case and the perturbed parameter $i$, respectively. (b) Relative invaded volume for the five most influential parameters for the different levels of chemotaxis. $\mathrm{V}_{i, \text { Low }}$ is the invaded volume for the perturbed parameter $i$ for the LC tumor.

the compact core of the tumor as migrating cells that have spread faster switch to the proliferating phenotype and populate the invaded tissue. Our results are in agreement with the results of Zhang et al. $^{79}$ where they determined that the expansion of a tumor is increased by the appearance of clones that are more sensitive to chemoattractant gradients. In a similar work, the effect of the search precision on the expansion rate of a tumor was also studied in ${ }^{88}$. They found that a high-search precision favors the expansion rate of the tumor although a maximal search precision results in a suboptimal invasion rate. Our results are also consistent with other results from a very different modeling approach. Using a continuum model, Wise et al. ${ }^{50}$ characterized the capacity of tumor cells to spread by an adhesion strength parameter, and found that low-adhesion strength (analogous to our highchemotaxis case) results in larger, more irregular tumors with the formation of buds driven by the redistribution of nutrients.

The environment also plays an important role on tumor morphology, growth dynamics and invasion rate. Our model predicts that the destruction of blood vessels (and, hence, the decrease of nutrient concentration) contributes to irregular morphologies for the medium- and high-chemotaxis cases. Similarly, using an agent-based model of a solid tumor, Gerlee and Anderson ${ }^{78}$ found that high concentrations of nutrients favor the formation of regular shaped tumors, whereas lower concentrations of nutrients lead to irregular morphologies. However, their simulations show that lower concentrations of nutrients correlate with lower growth rates (i.e., in terms of the number of cells), and have only a slight effect on the invasion rate (i.e., in terms of the invaded area) of the tumors. In contrast, our results suggest that vessel occlusion can induce higher invasion rates for tumor of $\mathrm{HC}$ cells even though it is accompanied by a drop on the nutrients level. The main reason for this difference is the presence of migrating cells in our model as opposed to their model which does not take these cells into account.

Winkler et al. ${ }^{89}$ developed an in vivo model to evaluate the effect of antiangiogenic therapy on the progression of angiogenesis dependent human glioma tumors. Antiangiogenic therapy induced a "normalization window" where aberrant tumor vasculature significantly recovered the structural and functional properties of the normal vasculature. During this window, tumor oxygenation increased, and tumor growth was briefly delayed. This coincides with our simulation results that predict slower tumor growth for tumors of HC cells when blood vessels are not destroyed (Figure 10). Although the growth delay observed in these experimental models may be due to several factors including changes in the expression of growth factors produced by tumor and tissue cells, it would be insightful to assess the contribution of chemotaxis on the tumor dynamics in such experimental settings.

We anticipate that a number of model simplifications can be ameliorated in future enhancements. At the tumor level, incorporation of other biological processes characteristic of glioblastomas such as tumor-induced angiogenesis, hypoxia, and production of metabolic waste will provide a more complete picture of tumor progression. The assumption of cellular migration driven exclusively by chemotaxis can be relaxed by considering adhesion molecules in the cells and the extracellular matrix, as well as the repulsing effect of metabolic waste. Similarly, the assumption of vessel occlusion as the only mechanism of vasculature remodeling will be relaxed by the incorporation of angiogenesis and other mechanisms such as increased leakiness that can be considered. At the cellular level, we assumed that the phenotype decision process is determined by the MAPK pathway. Although there is experimental evidence that correlates the activity of the MAPK pathway with migration and proliferation in gliomas, ${ }^{13}$ there is no quantitative information that links the activation levels of the MAPK pathway and output of the decision process. Therefore, we have used a phenomenological model that resembles in general terms the response of the rates of proliferation and migration of glioma cells to different concentrations of growth factors. ${ }^{80}$ Moreover, the EGFR activated intracellular signaling cascade would be better represented by considering the coactivation of more pathways, such as the MAPK, PI3K/Akt, and PLC $\gamma$ pathways. This network will provide a more complete picture of the proliferating vs. migrating decision process. We expect that these model enhancements will have a relevant effect on tumor progression. However, given the interdependence among these processes (and the ones already included in the model), the overall effect on the simulation results is difficult to assess a priori, but once the implementation of the incorporations is made the conclusions based on the current model can be revised. Contact inhibition is a major assumption 
tailored to the simulations using the regular grid. This assumption affects the distribution of migrating and proliferating cells by restricting cells in regions that have reached the maximum cellular density to be quiescent. This assumption can be relaxed by grid-free approach (e.g., cell location is determined by solving a minimum energy configuration problem), although at a higher computational expense. However, the effect on the invasion rate of the tumor is minor since the main contribution comes from migrating and proliferative activity at the outer rim of the tumor as evidenced by experimental observation. Another difficulty associated with grid-constraint models is the limitation to treat cell-cell mechanical interactions. This limitation could be alleviated by incorporating detailed tissue mechanics; however, efficient algorithms to overcome the associated computational burden need to be developed. We believe that the model and the simulation scheme proposed in this work can be the base of an ambitious modeling effort that include additional components to those mentioned, such as the influence of waste from necrotic cells and homotype chemoattraction.

\section{Acknowledgments}

The authors gratefully acknowledge funding from the Penn State Institute for CyberScience Seed Funding and the National Science Foundation (NSF-CAREER award \#CBET 06-44519).

\section{Literature Cited}

1. Stupp R, Mason WP, van den Bent MJ, Weller M, Fisher B, Taphoorn MJB, Belanger K, Brandes AA, Marosi C, Bogdahn U, Curschmann J, Janzer RC, Ludwin SK, Gorlia T, Allgeier A, Lacombe D, Cairncross JG, Eisenhauer E, Mirimanoff RO, Van Den Weyngaert D, Kaendler S, Krauseneck P, Vinolas N, Villa S, Wurm RE, Maillot MHB, Spagnolli F, Kantor G, Malhaire JP, Renard L, De Witte O, Scandolaro L, Vecht CJ, Maingon P, Lutterbach J, Kobierska A, Bolla M, Souchon R, Mitine C, Tzuk-Shina T, Kuten A, Haferkamp G, de Greve J, Priou F, Menten J, Rutten I, Clavere P, Malmstrom A, Jancar B, Newlands E, Pigott K, Twijnstra A, Chinot O, Reni M, Boiardi A, Fabbro M, Campone M, Bozzino J, Frenay M, Gijtenbeek J, Brandes AA, Delattre JY, Bogdahn U, De Paula U, van den Bent MJ, Hanzen C, Pavanato G, Schraub S, Pfeffer R, Soffietti R, Weller M, Kortmann RD, Taphoorn M, Torrecilla JL, Marosi C, Grisold W, Huget P, Forsyth P, Fulton D, Kirby S, Wong R, Fenton D, Fisher B, Cairncross G, Whitlock P, Belanger K, Burdette-Radoux S, Gertler S, Saunders S, Laing K, Siddiqui J, Martin LA, Gulavita S, Perry J, Mason W, Thiessen B, Pai H, Alam ZY, Eisenstat D, Mingrone W, Hofer S, Pesce G, Curschmann J, Dietrich PY, Stupp R, Mirimanoff RO, Thum P, Baumert B, Ryan G. Radiotherapy plus concomitant and adjuvant temozolomide for glioblastoma. N Engl J Med. 2005;352(10):987-996.

2. Kreisl TN, Kim L, Moore K, Duic P, Royce C, Stroud I, Garren N, Mackey M, Butman JA, Camphausen K, Park J, Albert PS, Fine HA. Phase II Trial of single-agent bevacizumab followed by bevacizumab plus irinotecan at tumor progression in recurrent glioblastoma. J Clin Oncol. 2008;16:3055.

3. Narayana A, Golfinos JG, Fischer I, Raza S, Kelly P, Parker E, Knopp EA, Medabalmi P, Zagzag D, Eagan P, Gruber ML. Feasibility of using bevacizumab with radiation therapy and temozolomide in newly diagnosed high-grade glioma. Int J Radiat Oncol Biol Phys. 2008;72(2):383-389.

4. Batchelor TT, Sorensen AG, di Tomaso E, Zhang WT, Duda DG, Cohen KS, Kozak KR, Cahill DP, Chen PJ, Zhu MW, Ancukiewicz M, Mrugala MM, Plotkin S, Drappatz J, Louis DN, Ivy P, Scadden DT, Benner T, Loeffler JS, Wen PY, Jain RK. AZD2171, a panVEGF receptor tyrosine kinase inhibitor, normalizes tumor vasculature and alleviates edema in glioblastoma patients. Cancer Cell. 2007;11(1):83-95.

5. Desjardins A, Reardon DA, Herndon JE, Marcello J, Quinn J, Rich JN, Sathornsumetee S, Gururangan S, Sampson J, Bailey L, Bigner
DD, Friedman AH, Friedman HS, Vredenburgh JJ. Bevacizumab plus irinotecan in recurrent WHO grade 3 malignant gliomas. Clinical Cancer Res. 2008;14(21):7068-7073.

6. Norden AD, Wen PY. Glioma therapy in adults. Neurologist. 2006; 12(6):279-292.

7. Furnari FB, Fenton T, Bachoo RM, Mukasa A, Stommel JM, Stegh A, Hahn WC, Ligon KL, Louis DN, Brennan C, Chin L, DePinho RA, Cavenee WK. Malignant astrocytic glioma: genetics, biology, and paths to treatment. Genes Develop. 2007;21(21):2683-2710.

8. Nakada M, Niska JA, Tran NL, McDonough WS, Berens ME. EphB2/R-Ras signaling regulates glioma cell adhesion, growth, and invasion. Am J Pathol. 2005;167(2):565-76.

9. Park CM, Park MJ, Kwak HJ, Lee HC, Kim MS, Lee SH, Park IC, Rhee $\mathrm{CH}$, Hong SI. Ionizing radiation enhances matrix metalloproteinase-2 secretion and invasion of glioma cells through Src/epidermal growth factor receptor-mediated p38/Akt and phosphatidylinositol 3-kinase/Akt signaling pathways. Cancer Res. 2006;66(17): 8511-8519.

10. Hoelzinger DB, Demuth T, Berens ME. Autocrine factors that sustain glioma invasion and paracrine biology in the brain microenvironment. J Nat Cancer Inst. 2007;99(21):1583-1593.

11. Frieboes HB, Zheng X, Sun CH, Tromberg B, Gatenby R, Cristini V. An integrated computational/experimental model of tumor invasion. Cancer Res. 2006;66(3):1597-1604.

12. Demuth T, Hopf NJ, Kempski O, Sauner D, Herr M, Giese A, Perneczky A. Migratory activity of human glioma cell lines in vitro assessed by continuous single cell observation. Clin Exp Metastasis. 2001;18(7):589-597.

13. Lind CR, Gray CW, Pearson AG, Cameron RE, O'Carroll SJ, Narayan PJ, Lim J, Dragunow M. The mitogen-activated/extracellular signal-regulated kinase kinase 1/2 inhibitor U0126 induces glial fibrillary acidic protein expression and reduces the proliferation and migration of C6 glioma cells. Neuroscience. 2006;141(4):1925-33.

14. Krueger JS, Keshamouni VG, Atanaskova N, Reddy KB. Temporal and quantitative regulation of mitogen-activated protein kinase (MAPK) modulates cell motility and invasion. Oncogene. 2001; 20(31):4209-4218.

15. Behin A, Hoang-Xuan K, Carpentier AF, Delattre JY. Primary brain tumours in adults. Lancet. 2003;361(9354):323-331.

16. Arwert E, Hingtgen S, Figueiredo JL, Bergquist H, Mahmood U, Weissleder R, Shah K. Visualizing the dynamics of EGFR activity and antiglioma therapies in vivo. Cancer Res. 2007;67(15):7335-7342.

17. Sakariassen PO, Prestegarden L, Wang J, Skaftnesmo KO, Mahesparan R, Molthoff C, Sminia P, Sundlisaeter E, Misra A, Tysnes BB, Chekenya M, Peters H, Lende G, Kalland KH, Oyan AM, Petersen K, Jonassen I, van der Kogel A, Feuerstein BG, Terzis AJA, Bjerkvig R, Enger PO. Angiogenesis-independent tumor growth mediated by stem-like cancer cells. Proc Nat Acad Sci USA. 2006;103(44):16466-16471.

18. Araujo RP, McElwain DL. A history of the study of solid tumour growth: The contribution of mathematical modelling. Bull Math Biol. 2004;66(5):1039-91.

19. Bellomo N, Li NK, Maini PK. On the foundations of cancer modelling: Selected topics, speculations, and perspectives. Math Models Methods Appl Sci. 2008;18(4):593-646.

20. Chaplain MA, McDougall SR, Anderson AR. Mathematical modeling of tumor-induced angiogenesis. Annu Rev Biomed Eng. 2006;8: 233-57.

21. Mantzaris NV, Webb S, Othmer HG. Mathematical modeling of tumor-induced angiogenesis. J Math Biology. 2004;49(2):111-187.

22. Hatzikirou H, Deutsch A, Schaller C, Simon M, Swanson K. Mathematical modelling of glioblastoma tumour development: A review. Math Models Methods Appl Sci. 2005;15(11):1779-1794.

23. Swanson KR, Bridge C, Murray JD, Alvord EC, Jr. Virtual and real brain tumors: using mathematical modeling to quantify glioma growth and invasion. J Neurol Sci. 2003;216(1):1-10.

24. Harpold HLP, Alvord EC, Swanson KR. The evolution of mathematical Modeling of glioma proliferation and invasion. J Neuropathol Exp Neurol. 2007;66(1):1-9.

25. Bhalla US. Models of cell signaling pathways. Curr Opin Genet Develop. 2004;14(4):375-381.

26. Hornberg JJ, Binder B, Bruggeman FJ, Schoeberl B, Heinrich R, Westerhoff HV. Control of MAPK signalling: from complexity to what really matters. Oncogene. 2005;24(36):5533-5542. 
27. Christopher R, Dhiman A, Fox J, Gendelman R, Haberitcher T, Kagle D, Spizz G, Khalil IG, Hill C. Data-driven computer simulation of human cancer cell. Ann NY Acad Sci. 2004;1020:132-153.

28. Kholodenko BN. Negative feedback and ultrasensitivity can bring about oscillations in the mitogen-activated protein kinase cascades. Eur J Biochem. 2000;267(6):1583-1588.

29. Boushaba K, Levine HA, Nilsen-Hamilton M. A mathematical model for the regulation of tumor dormancy based on enzyme kinetics. Bull Math Biol. 2006;68(7):1495-1526.

30. Arciero JC, Jackson TL, Kirschner DE. A mathematical model of tumor-immune evasion and siRNA treatment. Discrete Contin Dynam Syst Series B. 2004;4(1):39-58.

31. Abbott LH, Michor F. Mathematical models of targeted cancer therapy. Br J Cancer. 2006;95(9):1136-1141.

32. Johnston MD, Edwards CM, Bodmer WF, Maini PK, Chapman SJ. Mathematical modeling of cell population dynamics in the colonic crypt and in colorectal cancer. Proc Nat Acad Sci USA. 2007; 104(10):4008-4013.

33. Komarova NL, Wodarz D. Drug resistance in cancer: Principles of emergence and prevention. Pro Nat Acad Sci USA. 2005;102(27): 9714-9719.

34. Kirkby NF, Jefferies SJ, Jena R, Burnet NG. A mathematical model of the treatment and survival of patients with high-grade brain tumours. J Theor Biol. 2007;245(1):112-124.

35. Sole RV, Rodriguez-Caso C, Deisboeck TS, Saldana J. Cancer stem cells as the engine of unstable tumor progression. J Theor Biol. 2008;253(4):629-37.

36. Stein AM, Demuth T, Mobley D, Berens M, Sander LM. A mathematical model of glioblastoma tumor spheroid invasion in a threedimensional in vitro experiment. Biophys J. 2007;92(1):356-365.

37. Plank MJ, Sleeman BD, Jones PF. A mathematical model of tumour anglogenesis, regulated by vascular endothelial growth factor and the angiopoietins. J Theor Biol. 2004;229(4):435-454.

38. Peterson JW, Carey GF, Knezevic DJ, Murray BT. Adaptive finite element methodology for tumour angiogenesis modelling. Int $J$ Numer Methods Eng. 2007;69(6):1212-1238.

39. Mac Gabhann F, Ji JW, Popel AS. Computational model of vascular endothelial growth factor spatial distribution in muscle and proangiogenic cell therapy. PloS Comput Biol. 2006;2(9):1107-1120.

40. Dyson J, Sanchez E, Villella-Bressan R, Webb G. An age and spatially structured model of tumor invasion with haptotaxis. Discrete Contin Dynam Syst Series B. 2007;8(1):45-60.

41. Norris ES, King JR, Byrne HM. Modelling the response of spatially structured tumours to chemotherapy: Drug kinetics. Math Comput Modell. 2006;43(7-8):820-837.

42. Swanson KR, Alvord EC, Jr., Murray JD. Quantifying efficacy of chemotherapy of brain tumors with homogeneous and heterogeneous drug delivery. Acta Biotheor. 2002;50(4):223-37.

43. Powathil G, Kohandel M, Sivaloganathan S, Oza A, Milosevic M. Mathematical modeling of brain tumors: effects of radiotherapy and chemotherapy. Phys Med Biol. 2007;52(11):3291-3306.

44. Tee D, DiStefano J, 3rd. Simulation of tumor-induced angiogenesis and its response to anti-angiogenic drug treatment: mode of drug delivery and clearance rate dependencies. J Cancer Res Clin Oncol. 2004;130(1):15-24.

45. Ribba B, Saut O, Colin T, Bresch D, Grenier E, Boissel JP. A multiscale mathematical model of avascular tumor growth to investigate the therapeutic benefit of anti-invasive agents. J Theor Biol. 2006;243(4):532-41.

46. Khain E, Sander LM, Stein AM. A model for glioma growth. Complexity. 2005;11(2):53-57.

47. MacArthur BD, Please CP, Pettet GJ. A mathematical model of dynamic glioma-host interactions: receptor-mediated invasion and local proteolysis. Math Med Biol J Ima. 2005;22(3):247-264.

48. Levine HA, Pamuk S, Sleeman BD, Nilsen-Hamilton M. Mathematical modeling of capillary formation and development in tumor angiogenesis: Penetration into the stroma. Bull Math Biol. 2001; 63(5):801-863.

49. Ayati BP, Webb GF, Anderson ARA. Computational methods and results for structured multiscale models of tumor invasion. Multiscale Model Simul. 2006;5(1):1-20.

50. Wise SM, Lowengrub JS, Frieboes HB, Cristini V. Three-dimensional multispecies nonlinear tumor growth - I - Model and numerical method. J Theor Biol. 2008;253(3):524-543.
51. Anderson ARA, Weaver AM, Cummings PT, Quaranta V. Tumor morphology and phenotypic evolution driven by selective pressure from the microenvironment. Cell. 2006;127(5):905-915.

52. Alarcon T, Byrne HM, Maini PK. A multiple scale model for tumor growth. Multiscale Model Simul. 2005;3(2):440-475.

53. McDougall SR, Anderson ARA, Chaplain MAJ. Mathematical modelling of dynamic adaptive tumour-induced angiogenesis: Clinical implications and therapeutic targeting strategies. J Theor Biol. 2006;241(3):564-589.

54. Gevertz JL, Torquato S. Modeling the effects of vasculature evolution on early brain tumor growth. $J$ Theor Biol. 2006;243(4):517531.

55. Aubert M, Badoual M, Fereol S, Christov C, Grammaticos B. A cellular automaton model for the migration of glioma cells. Phys Biol. 2006;3(2):93-100.

56. Zhang L, Athale CA, Deisboeck TS. Development of a three-dimensional multiscale agent-based tumor model: Simulating gene-protein interaction profiles, cell phenotypes and multicellular patterns in brain cancer. $J$ Theor Biol. 2007;244(1):96-107.

57. Athale CA, Deisboeck TS. The effects of EGF-receptor density on multiscale tumor growth patterns. J Theor Biol. 2006;238(4):771-9.

58. Anderson ARA, Chaplain MAJ. Continuous and discrete mathematical models of tumor-induced angiogenesis. Bull Math Biol. 1998;60(5):857-899.

59. Zheng X, Wise SM, Cristini V. Nonlinear simulation of tumor necrosis, neo-vascularization and tissue invasion via an adaptive finiteelement/level-set method. Bull Math Biol. 2005;67(2):211-259.

60. Macklin P, Lowengrub J. Nonlinear simulation of the effect of microenvironment on tumor growth. J Theor Biol. 2007;245(4):677704.

61. Bauer AL, Jackson TL, Jiang Y. A cell-based model exhibiting branching and anastomosis during tumor-induced angiogenesis. Biophys $J$. 2007;92(9):3105-3121.

62. Jiang Y, Pjesivac-Grbovic J, Cantrell C, Freyer JP. A multiscale model for avascular tumor growth. Biophys J. 2005;89(6):38843894.

63. Stott EL, Britton NF, Glazier JA, Zajac M. Stochastic simulation of benign avascular tumour growth using the Potts model. Math Comput Modell. 1999;30(5-6):183-198.

64. Ambrosi D, Preziosi L. Cell adhesion mechanisms and stress relaxation in the mechanics of tumours. Biomech Model Mechanobiol. 2009;8(5):397-413.

65. Rejniak KA. An immersed boundary framework for modelling the growth of individual cells: An application to the early tumour development. J Theor Biol. 2007;247(1):186-204.

66. Hogea C, Davatzikos C, Biros G. An image-driven parameter estimation problem for a reaction-diffusion glioma growth model with mass effects. J Math Biol. 2008;56(6):793-825.

67. Frieboes HB, Lowengrub JS, Wise S, Zheng X, Macklin P, Bearer EL, Cristini V. Computer simulation of glioma growth and morphology. Neuroimage. 2007;37 Suppl.1:S59-70.

68. Teo CS, Tan WHK, Lee T, Wang CH. Transient interstitial fluid flow in brain tumors: Effect on drug delivery. Chem Eng Sci. 2005; 60(17):4803-4821.

69. Jellinger K. Glioblastoma multiforme - morphology and biology. Acta Neurochirurgica. 1978;42(1-2):5-32.

70. Nonaka H, Akima M, Hatori T, Nagayama T, Zhang Z, Ihara F. Microvasculature of the human cerebral white matter: arteries of the deep white matter. Neuropathology. 2003;23(2):111-8.

71. Welter M, Bartha K, Rieger H. Vascular remodelling of an arteriovenous blood vessel network during solid tumour growth. $J$ Theor Biol. 2009;259(3):405-22.

72. Hudetz AG. Mathematical model of oxygen transport in the cerebral cortex. Brain Res. 1999;817(1-2):75-83.

73. Bullitt E, Zeng DL, Gerig G, Aylward S, Joshi S, Smith JK, Lin WL, Ewend MG. Vessel tortuosity and brain tumor malignancy: A blinded study. Academic Radiol. 2005;12(10):1232-1240.

74. Bartha K, Rieger H. Vascular network remodeling via vessel cooption, regression and growth in tumors. $J$ Theor Biol. 2006;241(4): 903-918.

75. Zamecnik J, Vargova L, Homola A, Kodet R, Sykova E. Extracellular matrix glycoproteins and diffusion barriers in human astrocytic tumours. Neuropathol Appl Neurobiol. 2004;30(4):338-50. 
76. Freyer JP, Tustanoff E, Franko AJ, Sutherland RM. Insitu oxygenconsumption rates of cells in V-79 multicellular spheroids during growth. J Cellular Physiol. 1984;118(1):53-61.

77. Maly IV, Wiley HS, Lauffenburger DA. Self-organization of polarized cell signaling via autocrine circuits: Computational model analysis. Biophys J. 2004;86(1):10-22.

78. Gerlee P, Anderson ARA. A hybrid cellular automaton model of clonal evolution in cancer: The emergence of the glycolytic phenotype. J Theor Biol. 2008;250(4):705-722.

79. Zhang L, Strouthos CG, Wang Z, Deisboeck TS. Simulating brain tumor heterogeneity with a multiscale agent-based model: Linking molecular signatures, phenotypes and expansion rate. Math Comput Modell. 2009;49(1-2):307-319.

80. Giese A, Loo MA, Tran N, Haskett D, Coons SW, Berens ME. Dichotomy of astrocytoma migration and proliferation. Int $J$ Cancer. 1996;67(2):275-282.

81. Hegedus B, Czirok A, Fazekas I, B’Abel T, Madar'asz E, Vicsek T. Locomotion and proliferation of glioblastoma cells in vitro: statistical evaluation of videomicroscopic observations. $J$ Neurosurg. 2000;92(3):428-34.

82. Louis DN. Molecular pathology of malignant gliomas. Annu Rev Pathol. 2006;1:97-117.
83. Sander LM, Deisboeck TS. Growth patterns of microscopic brain tumors. Phys Rev E Stat Nonlin Soft Matter Phys. 2002;66(5 Pt 1): 051901.

84. Saad Y. Iterative Methods for Sparse Linear Systems. 2nd. ed. Philadelphia: SIAM; 2003.

85. Pope SB. Computationally efficient implementation of combustion chemistry using in situ adaptive tabulation. Combust Theory Modell. 1997;1(1):41-63.

86. Varshney A, Armaou A. Multiscale optimization using hybrid PDE/ kMC process systems with application to thin film growth. Chem Eng Sci. 2005;60(23):6780-6794.

87. Jain RK, Di Tomaso E, Duda DG, Loeffler JS, Sorensen AG, Batchelor TT. Angiogenesis in brain tumours. Nature Reviews Neuroscience. 2007;8(8):610-622.

88. Mansury Y, Deisboeck TS. The impact of "search precision" in an agent-based tumor model. J Theor Biol. 2003;224(3):325-37.

89. Winkler F, Kozin SV, Tong RT, Chae SS, Booth MF, Garkavtsev I, Xu L, Hicklin DJ, Fukumura D, di Tomaso E, Munn LL, Jain RK. Kinetics of vascular normalization by VEGFR2 blockade governs brain tumor response to radiation: Role of oxygenation, angiopoietin1, and matrix metal loproteinases. Cancer Cell. 2004;6(6):553-563.

Manuscript received Jan. 1, 2010, and revision received Apr. 17, 2010 